\title{
Les examens complémentaires prescrits aux urgences sont-ils souvent injustifiés et inutiles ?
}

\author{
Are the Complementary Exams Prescribed in Emergency Departments often Unjustified \\ and Unnecessary?
}

\section{E. Casalino}

Reçu le 13 décembre 2016; accepté le 13 décembre 2016

(C) SFMU et Lavoisier SAS 2016

Dans ce numéro des Annales Françaises de Médecine d'Urgences, Marjanovic et al. [1] rapportent une évaluation de la qualité de la prescription des examens complémentaires aux urgences. Cet article souligne l'importance de la rigueur dans la sélection des recommandations qui servent de référence à la mesure du respect des recommandations, décrit la méthode d'évaluation du respect des recommandations, et propose une mesure de l'impact, la durée de passage aux urgences. Les résultats de ce travail indiquent que le nonrespect des recommandations entraîne 57 \% de surprescriptions ou prescriptions non justifiées, donc inutiles. Cette surprescription est responsable d'un allongement significatif du temps total de passage aux urgences.

Peu de travaux ont évalué l'utilité ou la qualité de la prescription des examens biologiques [2] et radiologiques [3,4] aux urgences. Pourtant, nous pouvons estimer que dans les services d'urgences, au moins $30 \%$ des patients ont des examens biologiques et un autre $30 \%$ des examens radiologiques. Donc, $30 \%$ à $40 \%$ des patients ont au moins un examen complémentaire. Ces examens ont été associés à l'allongement du temps total de passage, indépendamment de l'âge des patients et de la décision finale d'hospitalisation $[5,6]$. L'allongement du temps total de passage aux urgences a été associé au risque de surcharge des urgences, de survenue d'effets secondaires graves, d'insatisfaction des patients et des personnels soignants, d'allongement de la durée d'hospitalisation et à un surcoût hospitalier [7]. Nous

E. Casalino $(\bowtie)$

Hôpital Bichat, 46, rue Henri-Huchard, F-75018 Paris, France

e-mail : enrique.casalino@aphp.fr

Université Paris-Diderot, Sorbonne-Paris-Cité, EA 7334

" Recherche clinique coordonnée ville-hôpital, méthodologies et société (REMES) », F-75010 Paris, France

Département des urgences (service d'accueil des urgences Bichat, service d'accueil des urgences Beaujon, Smur Beaujon), groupe hospitalier Paris Nord-Val-de-Seine, Paris, France pouvons donc estimer que la prescription injustifiée d'examens complémentaires peut être responsable de conséquences majeures sur l'organisation des urgences, la qualité et la sécurité des soins des patients et les conditions de travail des équipes soignantes.

Une limite dans l'évaluation de la qualité des prescriptions peut être que la comparaison entre les recommandations existantes pour certaines situations fréquentes permet de mettre en évidence des différences entre elles [8]. Par exemple, le nombre d'hémocultures, de flacons aéro- et anaérobies et le délai entre les prélèvements et le nombre d'hémocultures, sont mal précisés dans les recommandations des infections les plus fréquentes et même au cours du sepsis. Si la qualité de la prescription doit être évaluée sur son indication reconnue, comme ceci a été fait par l'équipe de Poitiers [1], elle peut l'être aussi sur le timing de cette prescription. Par exemple, dans la prise en charge du paludisme d'importation chez l'adulte, le test de diagnostic rapide ne doit être prescrit qu'après la réalisation de la goutte épaisse et seulement en cas de négativité de celle-ci.

Nous devons intégrer dans le projet qualité des services d'urgences la juste prescription des examens complémentaires. Nous pouvons établir, en accord avec les services et les laboratoires concernés, des règles de juste prescription des examens de biologie, et faire de même pour les examens de radiologie. Dans un projet qualité, cette transversalité de la décision est un gage de qualité et de réussite du projet. Si évaluer, comme ceci a été fait par l'équipe de Poitier [1], les intervalles qualité les plus courants aux urgences (temps de passage et délai d'attente médecin) semble évident, nous pourrons évaluer la réduction des délais d'attente pour l'obtention de l'examen et le rendu du résultat, la majoration de la satisfaction des patients, une réduction des coûts pour la structure et pour les patients. Ce projet qualité est cohérent avec une approche globale de réduction des délais d'attente, d'optimisation de la sécurité de la prise en charge des patients et d'amélioration de la satisfaction des patients. Il 
doit permettre également une amélioration des conditions de travail des équipes de soins, par exemple aux médecins d'éviter les discussions interminables sur les indications ou non de la radiologie en urgence, et aux équipes infirmières de réduire le risque d'accidents d'exposition au sang par des examens biologiques inutiles qui augmentent le délai entre la prescription d'un acte infirmier et sa réalisation effective. Nous avons tous à gagner à mettre en place une politique rigoureuse de juste prescription des examens de biologie et de radiologie !

Chacun doit jouer son rôle. Nous devons rappeler sans cesse aux jeunes internes mais aussi aux seniors l'importance de la juste prescription des examens complémentaires aux urgences, l'inutilité des bilans systématiques et non réfléchis, des examens « pour voir» ou basés sur " une fois j'ai vu un cas », et des examens en doublon (angioscanner pulmonaire positif pour une embolie et surprescription d'échodoppler veineux des membres inférieurs ou de scintigraphie pulmonaire). Chaque urgentiste est responsable de la qualité de ses prescriptions d'examens complémentaires et de la qualité des prescriptions de ses équipes.

Ce travail ouvre la porte à des nouvelles recherches d'évaluation de l'impact de stratégies de limitation de la prescription de certains examens dans des conditions cliniques spécifiques (hémocultures dans les pyélonéphrites ou dosage de la créatinine chez le sujet jeune avec une colique néphrétique), ou validation obligatoire par le senior de certains examens sur la base de scores cliniques prétest (angioscanner pour les embolies pulmonaires par exemple), ou à l'établissement de modèles de prescription d'examens ou de traitements en fonction du diagnostic évoqué et du terrain (place de la C-Reactive Proteine pour orienter la prescription des antibiotiques et des biomarqueurs dans la décision finale, par exemple).

\section{Références}

1. Marjanovic N, Mesrine M, Lardeur JY, et al (2017) Respects des recommandations de prescription des examens biologiques et évaluation de leur impact sur le temps de passage aux urgences. Ann Fr Med Urgence 7:7-15

2. Lapic I, Rogic D (2015) Laboratory utilization in the emergency department: are the requested tests patient-oriented? Signa Vitae 10:81-3

3. Radisphere (2013) Better radiology in the ED can reduce ED length of stay $10 \%$. Radisphere Radiology. http://www.radisphereradiology.com/resources/infographic/better-radiology-in-the-edcan-reduce-patient-los-by-10/ (Dernier accès le 5 décembre 2016)

4. Wong HJ, Sistrom CL, Benzer TI, et al (2013) Use of imaging in the emergency department: physicians have limited effect on variation. Radiology 268:779-89

5. Levine MB, Moore AB, Franck C, et al (2013) Variation in use of all types of computed tomography by emergency physicians. Am J Emerg Med 31:1437-42

6. Casalino E, Wargon M, Peroziello A, et al (2014) Predictive factors for longer length of stay in an emergency department: a prospective multicentre study evaluating the impact of age, patient's clinical acuity and complexity, and care pathways. Emerg Med J $31: 361-8$

7. Higginson I (2012) Emergency department crowding. Emerg Med J 29:437-43

8. US Department of Health and Human Services. Agency for Healthcare research and quality (2016) AHRQ's National Guideline Clearinghouse is a public resource for summaries of evidence-based clinical practice guidelines. https://www.guideline. gov/search?f_Clinical_Specialty=Emergency+Medicine\&fLockTerm=Emergency+Medicine (Dernier accès le 5 décembre 2016) 